\title{
Erratum to: Activation of the ventral tegmental area increased wakefulness in mice
}

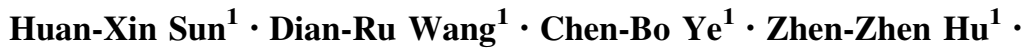

Chen-Yao Wang ${ }^{1} \cdot$ Zhi-Li Huang $^{1,2} \cdot$ Su-Rong Yang ${ }^{1}$

Published online: 4 August 2017

(C) Japanese Society of Sleep Research 2017

\section{Erratum to: Sleep Biol. Rhythms (2017) 15:107-115}

DOI 10.1007/s41105-017-0094-x

The original paper of this article unfortunately contained errors on Figure 1. The images (c-e) in right panel of Figure 1 are the same. The authors are sorry for these errors and certainly would like to correct the figure. Figure 1 is hereby corrected.

The online version of the original article can be found under doi:10.1007/s41105-017-0094-x.

\section{Su-Rong Yang}

sryang@shmu.edu.cn

1 Department of Pharmacology, School of Basic Medical Sciences, Fudan University, 138 Yixueyuan Road,

Box 229, Shanghai 200032, China

2 Institutes of Brain Science, Fudan University, Shanghai 200032, China 
Fig. 1 Expression of the mCherry, hM3Dq-mCherry, or hM4DimCherry in VTA.

$A$ Schematic representation of AAV vector microinjected. ITR, inverted terminal repeat; hSyn, human synapsin promoter; WPRE, woodchuck hepatitis virus post-transcriptional regulatory element. $B$ Red colored sites in coronal section show the injection target in mice. $C, D, E$ Coronal brain sections at the level of VTA prepared from mice expressing mCherry, hM3Dq-mCherry, or hM4Di-mCherry following hSyn-mCherry-AAV10 (C), hSynhM3Dq-AAV10 $(D)$, and hSyn-hM4Di -AAV10 (E) microinjection. " $c, d, e$ " higher magnification of the square region indicated in " $A$, $B, C$ ". Scale bars $=100 \mu \mathrm{m}$ $(C, D, E) ; 50 \mu \mathrm{m}(c, d, e)$

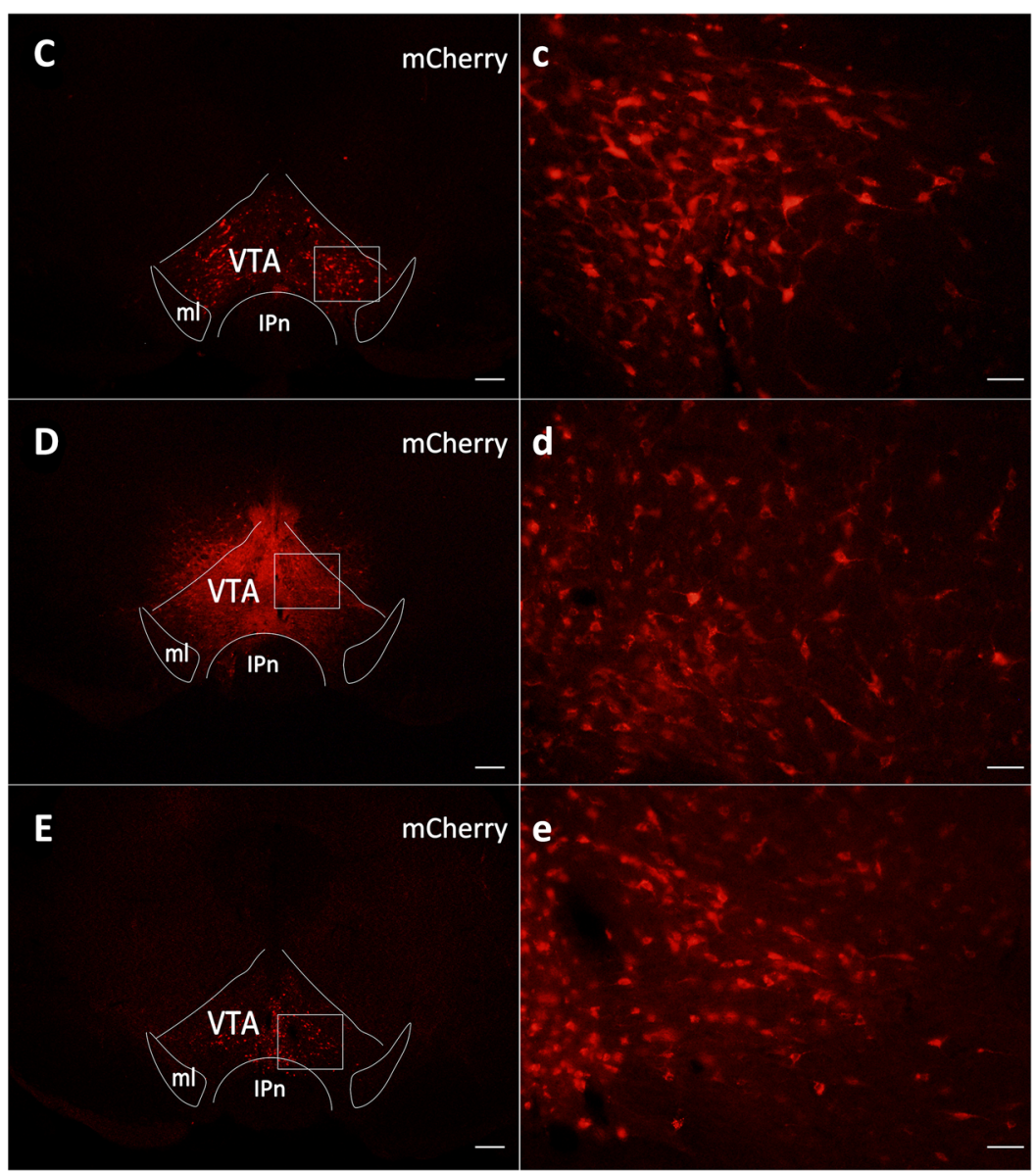

\section{Control}

hM3Dq

hM4Di 